\title{
COMPACTNESS OF A CONFORMAL BOUNDARY OF THE EUCLIDEAN UNIT BALL
}

\author{
Päivi Lammi \\ University of Jyväskylä, Department of Mathematics and Statistics \\ P.O. Box 35 (MaD), FI-40014 University of Jyväskylä, Finland; paivi.e.lammi@jyu.fi
}

\begin{abstract}
We study conformal metrics $d_{\rho}$ on the Euclidean unit ball $\mathbf{B}^{n}$. We assume that either the density $\rho$ associated with the metric $d_{\rho}$ satisfies a logarithmic volume growth condition for small balls or that $\rho$ satisfies a Harnack inequality and a suitable sub-Euclidean volume growth condition. We prove that the $\rho$-boundary $\partial_{\rho} \mathbf{B}^{n}$ is homeomorphic to $\mathbf{S}^{n-1}$ if and only if $\partial_{\rho} \mathbf{B}^{n}$ is compact. In the planar case, the compactness of $\partial_{\rho} \mathbf{B}^{2}$ is further equivalent to local connectivity of the $\rho$-boundary together with the boundedness of $\left(\mathbf{B}^{2}, d_{\rho}\right)$.
\end{abstract}

\section{Introduction}

Let $\Omega \subset \mathbf{R}^{2}$ be a bounded simply connected domain. A conformal map $f$ of the unit disc $\mathbf{B}^{2}$ onto $\Omega$ can be extended to a homeomorphism of $\overline{\mathbf{B}}^{2}$ onto $\bar{\Omega}$ if and only if $\Omega$ is a Jordan domain (cf. [Po]). Because $\Omega:=\mathbf{B}^{2} \backslash(\{0\} \times[0,1])$ is not Jordan, any conformal map from the unit disk onto $\Omega$ will not have a homeomorphic extension to the closed disk. If we equip $\Omega$ with the internal metric $d_{I}$ - that is the path metric in the Euclidean sense - then the homeomorphic extension onto $\bar{\Omega}_{I}$ can be accomplished, where $\bar{\Omega}_{I}$ is the metric completion of $\Omega$ in the sense of the internal metric $d_{I}$.

On the other hand, given a path $\gamma \subset \Omega$ and letting $\tilde{\gamma}=f^{-1} \circ \gamma$ we have that the Euclidean length of the path $\gamma$ is

$$
\ell(\gamma)=\int_{\gamma}|d z|=\int_{f(\tilde{\gamma})}|d z|=\int_{\tilde{\gamma}}\left|f^{\prime}(z)\right||d z| .
$$

Hence by defining a new metric $d_{\rho}$ on the unit disc $\mathbf{B}^{2}$,

$$
d_{\rho}(u, v)=\inf \int_{\hat{\gamma}}\left|f^{\prime}(z)\right||d z|,
$$

where the infimum is taken over all paths $\hat{\gamma}$ joining points $u, v \in \mathbf{B}^{2}$, we deduce that for each pair of points $x, y \in \Omega$,

$$
d_{I}(x, y)=d_{\rho}\left(f^{-1}(x), f^{-1}(y)\right) .
$$

Thus we can identify the metric spaces $\left(\Omega, d_{I}\right)$ and $\left(\mathbf{B}^{2}, d_{\rho}\right)$, and also their metric boundaries $\partial_{I} \Omega$ and $\partial_{\rho} \mathbf{B}^{2}$.

The density $\rho(z)=\left|f^{\prime}(z)\right|$ is a special case of the concept of a conformal deformation introduced by Bonk, Koskela and Rohde in [BKR]. A continuous density

doi:10.5186/aasfm.2011.3601

2010 Mathematics Subject Classification: Primary 30C65.

Key words: Boundary, compactness, conformal metrics.

The author was supported by the Vilho, Yrjö and Kalle Väisälä Fund, and the Academy of Finland, grant no. 120972. 
$\rho: \mathbf{B}^{n} \rightarrow(0, \infty)$ is conformal provided it satisfies two simple conditions (cf. conditions $\mathrm{HI}(\mathrm{A})$ and $\mathrm{VG}(\mathrm{B})$ below). The first condition requires that the density $\rho$ is roughly constant on Whitney type balls, and the second condition says that the measure $\mu_{\rho}$ of balls in the metric $d_{\rho}$ should not grow faster than the Euclidean volume of balls in $\mathbf{R}^{n}$. The measure $\mu_{\rho}$ is associated with $\rho$ in the usual way by using the volume element $\rho^{n} d m_{n}$. Recent papers on conformal deformations include [BKR], $[\mathrm{BHR}],[\mathrm{BK}],[\mathrm{Her}]$ and $[\mathrm{KL}]$. There are also some studies concerning the boundary behavior of conformal deformations, see [HKT], [NT1] and [NT2].

In this paper we give necessary and sufficient conditions on the density $\rho$ to guarantee that the metric boundary $\partial_{\rho} \mathbf{B}^{n}$ is homeomorphic to the sphere $\mathbf{S}^{n-1}$. We restrict our attention to densities $\rho$ whose associated measure $\mu_{\rho}$ has allowable growth; see Section 2 for precise details, and we prove that the compactness of the $\rho$-boundary $\partial_{\rho} \mathbf{B}^{n}$ implies that $\partial_{\rho} \mathbf{B}^{n}$ and $\mathbf{S}^{n-1}$ are homeomorphic.

Theorem 1.1. Let $\rho: \mathbf{B}^{n} \rightarrow(0, \infty)$ be a density whose associated measure $\mu_{\rho}$ has allowable growth. Then the following conditions are equivalent:

(i) $\left(\partial_{\rho} \mathbf{B}^{n}, d_{\rho}\right)$ is compact.

(ii) $\left(\mathbf{B}^{n} \cup \partial_{\rho} \mathbf{B}^{n}, d_{\rho}\right)$ is compact.

(iii) The identity map id: $\left(\mathbf{B}^{n}, d_{\rho}\right) \rightarrow\left(\mathbf{B}^{n},|\cdot|\right)$ has a homeomorphic extension to $i:\left(\mathbf{B}^{n} \cup \partial_{\rho} \mathbf{B}^{n}, d_{\rho}\right) \rightarrow\left(\mathbf{B}^{n} \cup \mathbf{S}^{n-1},|\cdot|\right)$.

In addition, when $n=2$ the above are also equivalent to

(iv) $\mathbf{B}_{\rho}^{n}$ is bounded and $\partial_{\rho} \mathbf{B}^{n}$ is locally connected.

However, when $n \geq 3$, there are examples where (iv) holds but none of (i,ii,iii) are true.

The proof of Theorem 1.1 is in Section 4, and in Example 4.1 we show that condition (iv) is not sufficient in higher dimensions.

This Theorem 1.1 is new even when $\mu_{\rho}$ has Euclidean growth. Moreover, some growth condition on $\mu_{\rho}$ is necessary because otherwise it can happen that $\partial_{\rho} \mathbf{B}^{n}$ is compact but $\operatorname{diam}_{\rho}\left(\partial_{\rho} \mathbf{B}^{n}\right)=0$ (cf. $\left.[\mathrm{HKT}]\right)$.

\section{Definitions}

We denote by $|x|$ the Euclidean norm of $x \in \mathbf{R}^{n}$, by $|x-y|$ the Euclidean distance between two points $x, y \in \mathbf{R}^{n}$, by $\operatorname{diam}(E)$ the diameter of a set $E \subset \mathbf{R}^{n}$ and by $d(E, F)=\inf \{|x-y|: x \in E, y \in F\}$ the distance between two sets $E, F \subset \mathbf{R}^{n}$. Furthermore, $B^{n}(a, r)=\left\{x \in \mathbf{R}^{n}:|a-x|<r\right\}$ for $a \in \mathbf{R}^{n}$ and $r>0$ is the open ball with centre $a$ and radius $r$ and $S^{n-1}(a, r)=\left\{x \in \mathbf{R}^{n}:|a-x|=r\right\}$ for $a \in \mathbf{R}^{n}$ and $r>0$ is the sphere with centre $a$ and radius $r$. We omit the subscript $n$ if the dimension of the ball or the sphere is clear. We abbreviate the unit ball $B^{n}(0,1)$ to $\mathbf{B}^{n}$ and the Euclidean boundary of $\mathbf{B}^{n}$ to $\mathbf{S}^{n-1}$.

Let $(\Omega,|\cdot|) \subset \mathbf{R}^{n}$ be a domain. The boundary $\partial \Omega$ of $\Omega$ is $\partial \Omega:=\bar{\Omega} \backslash \Omega$, where $\bar{\Omega}$ is the metric completion of $\Omega$. A curve in $\Omega$ is a continuous mapping $\gamma: I \rightarrow \Omega$ from an interval $I \subset \mathbf{R}$ to $\Omega$. The interval $I$ may be open, half-open, closed, bounded or unbounded. We identify the curve $\gamma$ and the image set $\gamma(I)$ by denoting both by $\gamma$. We denote by $\ell(\gamma) \in[0, \infty]$ the Euclidean length of $\gamma$. If $\ell(\gamma)<\infty$, then the curve is called rectifiable. The curve $\gamma$ is locally rectifiable if $\ell\left(\left.\gamma\right|_{[a, b]}\right)<\infty$ for every closed interval $[a, b] \subset I$. 
In this paper, we assume that the parameter interval $I$ is $[0,1]$ or $[0,1)$. The inverse of a curve $\gamma:[0,1] \rightarrow \Omega$ is the curve $\bar{\gamma}:[0,1] \rightarrow \Omega, \bar{\gamma}(t)=\gamma(1-t)$. If $\gamma:[0,1) \rightarrow \Omega$ is rectifiable, then there is a continuous extension $\gamma:[0,1] \rightarrow \bar{\Omega}$, and thus $\gamma$ has a terminal endpoint $\gamma(1):=\lim _{t>1} \gamma(t) \in \bar{\Omega}$. If $\gamma_{1}, \gamma_{2}:[0,1] \rightarrow \Omega$ are curves such that $\gamma_{1}(1)=\gamma_{2}(0)$, then we define a concatenation $\gamma:=\gamma_{1} \star \gamma_{2}:[0,1] \rightarrow \Omega$ by setting

$$
\gamma(t)= \begin{cases}\gamma_{1}(2 t), & \text { when } 0 \leq t \leq \frac{1}{2} \\ \gamma_{2}(2 t-1), & \text { when } \frac{1}{2}<t \leq 1\end{cases}
$$

Let $\rho: \Omega \rightarrow(0, \infty)$ be a continuous function, a density. The $\rho$-length of a locally rectifiable curve $\gamma$ in $\Omega$ is

$$
\ell_{\rho}(\gamma)=\int_{\gamma} \rho(x)|d x|
$$

where $|d x|$ denotes integration with respect to Euclidean length. If $\ell_{\rho}(\gamma)<\infty$ we say that the curve $\gamma$ is $\rho$-rectifiable. Furthermore, the $\rho$-metric

$$
d_{\rho}(x, y)=\inf _{\gamma} \ell_{\rho}(\gamma) \quad \text { for } x, y \in \Omega,
$$

where the infimum is taken over all curves $\gamma$ in $\Omega$ joining points $x$ and $y$, is welldefined. For clarity, the usual metric notations which refer to the metric $d_{\rho}$ will have the additional subscript $\rho$. For example, $\operatorname{diam}_{\rho}(E)$ is the diameter of a set $E$ in the metric $d_{\rho}$. We also abbreviate the metric space $\left(\Omega, d_{\rho}\right)$ to $\Omega_{\rho}$.

The $\rho$-boundary $\partial_{\rho} \Omega$ of $\Omega_{\rho}$ can be defined by standard constructions using Cauchy sequences. An equivalent definition for $\partial_{\rho} \Omega$ is $\partial_{\rho} \Omega:=\bar{\Omega}_{\rho} \backslash \Omega_{\rho}$, where $\bar{\Omega}_{\rho}$ is the metric completion of $\Omega_{\rho}$.

For $\rho$-rectifiable curves $\alpha, \beta:[0,1) \rightarrow \Omega$,

$$
\alpha \sim \beta \Longleftrightarrow \lim _{t \nearrow 1} d_{\rho}(\alpha(t), \beta(t))=0
$$

defines an equivalence relation. We may also define the $\rho$-boundary $\partial_{\rho} \Omega$ as the set of equivalence classes of $\rho$-rectifiable curves $\gamma \subset \Omega_{\rho}$ that do not have an endpoint $\gamma(1)$ in $\Omega_{\rho}$. We leave the proof to the reader. In some cases this characterisation of the $\rho$-boundary $\partial_{\rho} \Omega$ is more useful than the standard definition.

When $\rho \equiv 1$, we obtain a special case of the metric $d_{\rho}$, which we denote by $d_{I}$ and call the inner metric. Notice that $d_{I}$ is the Euclidean path metric. We abbreviate $\left(\Omega, d_{I}\right)$ to $\Omega_{I}$ and call the boundary $\partial_{\rho} \Omega=\partial_{\mathrm{I}} \Omega$ the inner boundary.

We restrict our attention to the unit ball $\mathbf{B}^{n}$ and its deformations $\mathbf{B}_{\rho}^{n}$ for the rest of the paper. A continuous and positive function $\rho$ on $\mathbf{B}^{n}$ is called a conformal density (cf. [BKR]) if it satisfies both a Harnack type inequality $\mathrm{HI}(\mathrm{A})$ for some constant $A \geq 1$ :

$\mathrm{HI}(\mathrm{A}) \quad \frac{1}{A} \leq \frac{\rho(x)}{\rho(y)} \leq A \quad$ for all $x, y \in B(z,(1-|z|) / 2)$ and all $z \in \mathbf{B}^{n}$

and a volume growth condition $\mathrm{VG}(\mathrm{B})$ for some constant $B>0$ :

$\mathrm{VG}(\mathrm{B}) \quad \mu_{\rho}\left(B_{\rho}(x, r)\right) \leq B r^{n} \quad$ for all $x \in \mathbf{B}^{n}$ and $r>0$.

Here $\mu_{\rho}$ is the Borel measure on $\mathbf{B}^{n}$ defined by

$$
\mu_{\rho}(E)=\int_{E} \rho^{n} d m_{n} \quad \text { for a Borel set } E \subset \mathbf{B}^{n},
$$


where $m_{n}$ denotes $n$-dimensional Lebesgue measure.

In this paper we relax these strong conditions, $\mathrm{HI}(\mathrm{A})$ and $\mathrm{VG}(\mathrm{B})$, on the density $\rho$ but still need some volume growth to prove our theorems. A (general) volume growth condition $\mathrm{VG}$ is

$\mathrm{VG}$

$$
\mu_{\rho}\left(B_{\rho}(x, r)\right) \leq \varphi(r) \quad \text { for all } x \in \mathbf{B}^{n} \text { and } r>0,
$$

where $\varphi$ is an increasing homeomorphism of $(0, \infty)$. In this paper we consider especially a couple of particular conditions. If $\rho$ satisfies VG, where $\varphi(r)=D r^{n}|\log r|^{n-1}$ for some $D>0$ and for every $0<r<e^{-1}$, then we say that $\rho$ satisfies the (logarithmic) volume growth condition $\log -\mathrm{VG}$. We also say that $\mu_{\rho}$ has allowable growth if the density $\rho$ satisfies $\log -\mathrm{VG}$ or the density $\rho$ satisfies both conditions $\mathrm{HI}(\mathrm{A})$ and $\mathrm{VG}$, where $\varphi(r)=B r^{n-\epsilon}$ for some $B>0$ and $0<\epsilon<\frac{1}{1+\log A}$.

Let $\Gamma$ be a family of locally rectifiable curves in $\mathbf{B}^{n}$. The $\operatorname{modulus} \bmod \Gamma \in[0, \infty]$ is defined by

$$
\bmod \Gamma=\inf _{\tilde{\rho}} \int_{\mathbf{B}^{n}} \tilde{\rho}^{n} d m_{n}
$$

where the infimum is taken over all Borel measurable densities $\tilde{\rho}: \mathbf{B}^{n} \rightarrow[0, \infty]$ that are admissible. The density $\tilde{\rho}$ is admissible if $\ell_{\tilde{\rho}}(\gamma) \geq 1$ for every $\gamma \in \Gamma$. We denote by $\bmod \left(E, F ; \mathbf{B}^{n}\right)$ the modulus of the family of all rectifiable curves in $\mathbf{B}^{n}$ which connect subsets $E$ and $F$ of $\overline{\mathbf{B}^{n}}$. The curve $\gamma$ connects $E$ and $F$ if the closure of $\gamma$ intersects both $E$ and $F$. If $E$ and $F$ are disjoint continua in $\mathbf{B}^{n}$, that is, non-degenerate, compact and connected sets, then

$$
\phi_{n}(t) \leq \bmod \left(E, F ; \mathbf{B}^{n}\right),
$$

where

$$
t=\frac{\operatorname{dist}(E, F)}{\min \{\operatorname{diam}(E), \operatorname{diam}(F)\}}
$$

and

$$
\phi_{n}(t)=\frac{\omega_{n-1}}{2[\log (\lambda(1+t))]^{n-1}} .
$$

Here $\omega_{n-1}$ is the surface area of $\mathbf{S}^{n-1}$ and $\lambda=\lambda(n) \geq 1$ is a constant depending on $n$, see $[\mathrm{Ge}, \mathrm{Va}]$. We will also need the fact that $\bmod \left(E, F ; \mathbf{B}^{n}\right)=\infty$ whenever $E$ and $F$ are connected non-degenerate sets in $\overline{\mathbf{B}}^{n}$ whose closures have non-empty intersection. See, for example, [Va] for the basic properties of the modulus.

One can generalize these concepts to the setting of families of locally rectifiable curves which lie on a sphere $S^{n-1}(a, r)$ for some $a \in \mathbf{R}^{n}$ and $r>0$. If $\Gamma$ is a curve family on $S^{n-1}(a, r)$, then the modulus of $\Gamma$ with respect to $S^{n-1}(a, r)$ is defined by

$$
\bmod ^{S^{n-1}(a, r)}(\Gamma)=\inf _{\tilde{\rho}} \int_{S^{n-1}(a, r)} \tilde{\rho}^{n} d m_{n-1},
$$

where the infimum is taken over all Borel measurable densities $\tilde{\rho}: S^{n-1}(a, r) \rightarrow[0, \infty]$ for which $\ell_{\tilde{\rho}}(\gamma) \geq 1$ for every $\gamma \in \Gamma$. Here the integration is with respect to $n-1$ dimensional Lebesgue measure $m_{n-1}$ on $S^{n-1}(a, r)$.

From [Va, Theorem 10.2] we obtain a lower bound for the modulus $\bmod ^{S^{n-1}(a, r)}(E$, $F ; K)$ of the family of all rectifiable curves which connect disjoint non-empty subsets $E$ and $F$ of $\bar{K} \subset S^{n-1}(a, r)$. Here $K$ is a cap, which is an intersection of the sphere 
$S^{n-1}(a, r)$ and an open half space in $\mathbf{R}^{n}$, and $\bar{K}$ means the closure of $K$. The estimate [Va, Theorem 10.2] says that

$$
\bmod ^{S^{n-1}(a, r)}(E, F ; K) \geq \frac{C}{r},
$$

where $C=C(n)>0$ is a constant.

\section{Proofs of lemmas}

Let us start with one technical lemma, where we compute an upper bound for the modulus of certain curve families. This lemma is an analogue of Lemma $3.2 \mathrm{in}$ [BKR].

Lemma 3.1. Let $0<\delta \leq \frac{1}{16}$. Let $\rho$ be a continuous and positive density on $\mathbf{B}^{n}$ that satisfies $\log -V G$ at some point $x_{0} \in E \subset \mathbf{B}^{n}$, where $E$ is a non-empty set with $\operatorname{diam}_{\rho}(E) \leq \delta$. Let $\Gamma$ be the family of curves $\gamma$ in $\mathbf{B}^{n}$ that have one endpoint in $E$ and that the $\rho$-distance of the other endpoint from $E$ is at least $\frac{1}{2}$. Then

$$
\bmod (\Gamma) \leq 25^{n} D\left(\frac{2}{\log \left(\frac{|\log \delta|}{3 \log 2}\right)}\right)^{n-1}
$$

Proof. Let $\gamma \in \Gamma$ be arbitrary. We may assume that $\gamma(0) \in E$ and $d_{\rho}(\gamma(1), E) \geq$ $\frac{1}{2}$. Let $x_{0} \in E$ be a point as in our assumption. Let $j_{0} \in \mathbf{N}$ be a number such that $2^{-\left(j_{0}+1\right)}<\delta \leq 2^{-j_{0}}$. Let $A_{j}=B_{\rho}\left(x_{0}, 2^{-j}\right) \backslash B_{\rho}\left(x_{0}, 2^{-(j+1)}\right), j=0,1, \ldots, j_{0}-1$ and $A_{j_{0}}=B_{\rho}\left(x_{0}, 2^{-j_{0}}\right)$. Now $E \subset A_{j_{0}}$.

Define $\tilde{\rho}: \mathbf{B}^{n} \rightarrow[0, \infty)$,

$$
\tilde{\rho}(x)= \begin{cases}\frac{4 \log 2}{\left(\log \left(\frac{|\log \delta|}{3 \log 2}\right)\right)\left(\delta+d_{\rho}(x, E)\right)\left|\log \left(\delta+d_{\rho}(x, E)\right)\right|} \rho(x), & \text { when } d_{\rho}(x, E)<\frac{1}{e}-\delta, \\ 0, & \text { elsewhere. }\end{cases}
$$

Because $\gamma \cap A_{0} \neq \emptyset$ and $\tilde{\rho}(x)>0$ for every $x \in B_{\rho}\left(x_{0}, 2^{-2}\right)$, we have

$$
\begin{aligned}
\ell_{\tilde{\rho}}(\gamma) & \geq \frac{4 \log 2}{\log \left(\frac{|\log \delta|}{3 \log 2}\right)} \sum_{j=2}^{j_{0}} \int_{\gamma \cap A_{j}} \frac{\rho(x)}{\left(\delta+d_{\rho}(x, E)\right)\left|\log \left(\delta+d_{\rho}(x, E)\right)\right|} d s \\
& \geq \frac{4 \log 2}{\log \left(\frac{|\log \delta|}{3 \log 2}\right)} \sum_{j=2}^{j_{0}-1} \int_{\gamma \cap A_{j}} \frac{\rho(x)}{\left(\delta+2^{-j}\right) \mid \log 2^{-(j+1) \mid}} d s \\
& \geq \frac{4 \log 2}{\log \left(\frac{|\log \delta|}{3 \log 2}\right)} \sum_{j=2}^{j_{0}-1} \frac{2^{-(j+1)}}{2^{-j+1} \log \left(2^{j+1}\right)} \\
& \geq \frac{1}{\log \left(\frac{|\log \delta|}{3 \log 2}\right)} \sum_{j=2}^{j_{0}-1} \frac{1}{j+1} \geq \frac{1}{\log \left(\frac{|\log \delta|}{3 \log 2}\right)} \log \left(\frac{j_{0}+1}{3}\right) \\
& \geq \frac{1}{\log \left(\frac{|\log \delta|}{3 \log 2}\right)} \log \left(\frac{|\log \delta|}{3 \log 2}\right)=1 .
\end{aligned}
$$

In the last inequality we used the definition of $j_{0}$. 
Thus

$$
\begin{aligned}
& \bmod (\Gamma) \leq \int_{\mathbf{B}^{n}} \tilde{\rho}^{n} d m_{n} \\
& \leq\left(\frac{4 \log 2}{\log \left(\frac{|\log \delta|}{3 \log 2}\right)}\right)^{n}\left[\sum_{j=1}^{j_{0}} \int_{A_{j}} \frac{\rho(x)^{n}}{\left(\delta+d_{\rho}(x, E)\right)^{n}\left|\log \left(\delta+d_{\rho}(x, E)\right)\right|^{n}} d x\right] \\
& \leq\left(\frac{4 \log 2}{\log \left(\frac{|\log \delta|}{3 \log 2}\right)}\right)^{n}\left[\sum_{j=2}^{j_{0}} \int_{A_{j}} \frac{\rho(x)^{n}}{2^{-(j+1) n}\left|\log \left(\delta+2^{-j}\right)\right|^{n}} d x+\int_{A_{1}} \frac{\rho(x)^{n}}{2^{-2 n}\left|\log e^{-1}\right|^{n}} d x\right] \\
& \stackrel{\log -\mathrm{VG}}{\leq} D\left(\frac{4 \log 2}{\log \left(\frac{|\log \delta|}{3 \log 2}\right)}\right)^{n}\left[\sum_{j=2}^{j_{0}} \frac{2^{-j n}\left|\log \left(2^{-j}\right)\right|^{n-1}}{2^{-(j+1) n}\left|\log \left(2^{-j+1}\right)\right|^{n}}+\frac{e^{-n}\left|\log e^{-1}\right|^{n-1}}{2^{-2 n}}\right] \\
& \leq \frac{D}{\log 2}\left(\frac{8 \log 2}{\log \left(\frac{|\log \delta|}{3 \log 2}\right)}\right)^{n}\left[\sum_{j=2}^{j_{0}} \frac{j^{n-1}}{(j-1)^{n}}+\log 2\right] \\
& \text { (i) } \frac{2^{n-1} D}{\log 2}\left(\frac{8 \log 2}{\log \left(\frac{|\log \delta|}{3 \log 2}\right)}\right)^{n}\left[\sum_{j=2}^{j_{0}} \frac{1}{j-1}+1\right] \\
& \leq \frac{D}{\log 2}\left(\frac{16 \log 2}{\log \left(\frac{|\log \delta|}{3 \log 2}\right)}\right)^{n} \log \left(j_{0}-1\right) \leq \frac{D}{\log 2}\left(\frac{16 \log 2}{\log \left(\frac{|\log \delta|}{3 \log 2}\right)}\right)^{n} \log \left(\frac{|\log \delta|}{\log 2}\right) \\
& \stackrel{\text { (ii) }}{\leq} 5 D 16^{n}\left(\frac{\log 2}{\log \left(\frac{|\log \delta|}{3 \log 2}\right)}\right)^{n-1} \leq 25^{n} D\left(\frac{2}{\log \left(\frac{|\log \delta|}{3 \log 2}\right)}\right)^{n-1} \text {. }
\end{aligned}
$$

Above we used the facts

(i) $\left(\frac{j}{j-1}\right)^{n-1} \leq 2^{n-1}$ for every $j=2,3, \ldots$,

(ii) $\log \left(\frac{|\log \delta|}{\log 2}\right) \leq 5 \log \left(\frac{|\log \delta|}{3 \log 2}\right)$, when $\delta \leq \frac{1}{16}$.

In the previous lemma we assumed that the logarithmic growth condition log-VG holds only at one point in $E$ and only for small $\rho$-balls, meaning radius less than $e^{-1}$. For larger balls we actually do not need any growth condition.

Through Lemma 3.1 we obtain some information about the relation between the boundaries $\partial_{\rho} \mathbf{B}^{n}$ and $\mathbf{S}^{n-1}$.

Lemma 3.2. Let $\rho$ be a density that satisfies the volume growth condition log$V G$. Then every $d_{\rho}$-Cauchy sequence is also a Euclidean Cauchy sequence. In particular, for each $\xi \in \partial_{\rho} \mathbf{B}^{n}$ there is a unique natural identification point $\hat{\xi} \in \mathbf{S}^{n-1}$.

Proof. Let $\left(x_{k}\right) \subset \mathbf{B}^{n}$ be a $d_{\rho}$-Cauchy sequence. We may assume that $d_{\rho}\left(x_{k}, x_{k+1}\right)$ $\leq 2^{-k}$. Now we can construct a curve $\gamma:[0,1) \rightarrow \mathbf{B}^{n}$ such that for every $k \in \mathbf{N}$, $\gamma\left(t_{k}\right)=x_{k}$ for some $t_{k} \in[0,1), t_{k}<t_{k+1}, t_{k} \nearrow 1$ as $k \rightarrow \infty$, and $\ell_{\rho}\left(\left.\gamma\right|_{\left[t_{k}, t_{k+1}\right]}\right) \leq$ $2 d_{\rho}\left(x_{k}, x_{k+1}\right)$. Let us denote $\gamma_{k}:=\left.\gamma\right|_{\left[t_{k}, 1\right)}$. Then $\operatorname{diam}_{\rho}\left(\gamma_{k}\right) \rightarrow 0$, when $k \rightarrow \infty$.

We may assume that $\operatorname{diam}_{\rho}\left(\mathbf{B}^{n}\right) \geq 2$. If not, we choose $\tilde{\rho}=\frac{2}{\operatorname{diam}_{\rho}\left(\mathbf{B}^{n}\right)} \rho$ and do the following calculations with $\tilde{\rho}$. There is $N \in \mathbf{N}$ such that $\operatorname{diam}_{\rho}\left(\gamma_{k}\right)<\frac{1}{16}$ for every $k>N$. There is also a continuum $F \subset \mathbf{B}^{n}$ which contains more than one point and 
satisfies $d_{\rho}\left(\gamma_{k}, F\right) \geq \frac{1}{2}$ for every $k>N$. Then by Lemma 3.1

$$
\bmod \left(\gamma_{k}, F ; \mathbf{B}^{n}\right) \leq 25^{n} D\left(\frac{2}{\log \left(\frac{\left|\log \operatorname{diam} \rho\left(\gamma_{k}\right)\right|}{3 \log 2}\right)}\right)^{n-1} .
$$

On the other hand, we have $0<\operatorname{diam}(F)=: c \leq 2$ and so

$$
\tilde{t}=\frac{\operatorname{dist}\left(\gamma_{k}, F\right)}{\min \left\{\operatorname{diam}\left(\gamma_{k}\right), \operatorname{diam}(F)\right\}} \leq \frac{2}{\frac{c \operatorname{diam}\left(\gamma_{k}\right)}{2}}=\frac{4}{c \operatorname{diam}\left(\gamma_{k}\right)} .
$$

Hence from (2.1) we obtain

$$
\bmod \left(\gamma_{k}, F ; \mathbf{B}^{n}\right) \geq \frac{\frac{1}{2} \omega_{n-1}}{\left(\log \left(\lambda\left(1+\frac{4}{c \operatorname{diam}\left(\gamma_{k}\right)}\right)\right)\right)^{n-1}} .
$$

Since $\lim _{k \rightarrow \infty} \operatorname{diam}_{\rho}\left(\gamma_{k}\right)=0$, we conclude that both (RHS) and (LHS) go to zero and therefore $\lim _{k \rightarrow \infty} \operatorname{diam}\left(\gamma_{k}\right)=0$. Thus $\left(x_{k}\right)$ is a Euclidean Cauchy sequence.

Consequently, each $d_{\rho}$-Cauchy sequence, which converges to a given point $\xi \in$ $\partial_{\rho} \mathbf{B}^{n}$, is also a Euclidean Cauchy sequence, and the limit of these Euclidean Cauchy sequences must be same. Thus the identification point $\hat{\xi} \in \mathbf{S}^{n-1}$ is unique.

Hence, under the certain condition of $\rho$ every point in the $\rho$-boundary $\partial_{\rho} \mathbf{B}^{n}$ has a natural identification point in $\mathbf{S}^{n-1}$. What about the other way around? When does each point in $\mathbf{S}^{n-1}$ have a natural identification point in $\partial_{\rho} \mathbf{B}^{n}$ ? Our next lemma gives a simple condition under which for each $\hat{\xi} \in \mathbf{S}^{n-1}$ there exists a curve $\gamma$ in $\mathbf{B}^{n}$ that ends up $\hat{\xi}$ in the Euclidean sense, is $\rho$-rectifiable, and whose endpoint is in the $\rho$-boundary $\partial_{\rho} \mathbf{B}^{n}$ in the $d_{\rho}$-metric.

Lemma 3.3. Let $\rho$ be a density such that $\rho \in L^{n}\left(\mathbf{B}^{n}\right)$ and assume that $\mathbf{B}_{\rho}^{n}$ is bounded. Then for each $\hat{\xi} \in \mathbf{S}^{n-1}$ there exists a curve $\gamma:[0,1) \rightarrow \mathbf{B}^{n}$ with

$$
\ell_{\rho}(\gamma)<\infty, \lim _{t \nearrow 1}|\gamma(t)-\hat{\xi}|=0 \text { and } \xi:=\lim _{t \nearrow 1} \gamma(t) \in \partial_{\rho} \mathbf{B}^{n}
$$

moreover, the $\rho$-boundary point $\xi$ is uniquely determined by $\hat{\xi}$ and does not depend on the curve $\gamma$.

Proof. Suppose that $\operatorname{diam}_{\rho}\left(\mathbf{B}^{n}\right)<\infty$ and let $\hat{\xi} \in \mathbf{S}^{n-1}$. Let us construct a curve which has the desired properties.

Let $A_{j}=\left(B\left(\hat{\xi}, 2^{-j}\right) \backslash \bar{B}\left(\hat{\xi}, 2^{-(j+1)}\right)\right) \cap \mathbf{B}^{n}, j=1,2, \ldots$, be a Euclidean 'semi'-ring in $\mathbf{B}^{n}$. Because $\rho \in L^{n}\left(\mathbf{B}^{n}\right)$, we have that

$$
\int_{A_{j}} \rho^{n} d m_{n} \rightarrow 0, \quad \text { when } j \rightarrow \infty
$$

Let us first assume that $n=2$. Then we obtain by Hölder's inequality that

$$
\int_{A_{j}} \rho d m_{2} \leq 2^{-j} \sqrt{\pi}\left(\int_{A_{j}} \rho^{2} d m_{2}\right)^{\frac{1}{2}} \text { for every } j \in \mathbf{N} \text {. }
$$

On the other hand

$$
\int_{A_{j}} \rho d m_{2}=\int_{] 2^{-(j+1)}, 2^{-j}[} \int_{S^{1}(\hat{\xi}, t) \cap \mathbf{B}^{2}} \rho d s d t .
$$


Hence there exists $\left.t_{j} \in\right] 2^{-(j+1)}, 2^{-j}[$ such that

$$
\int_{A_{j}} \rho d m_{2} \geq \int_{] 2^{-(j+1)}, 2^{-j}[} \int_{S^{1}\left(\hat{\xi}, t_{j}\right) \cap \mathbf{B}^{2}} \rho d s d t=2^{-(j+1)} \int_{S^{1}\left(\hat{\xi}, t_{j}\right) \cap \mathbf{B}^{2}} \rho d s,
$$

and by inequality (3.1)

$$
\int_{S^{1}\left(\hat{\xi}, t_{j}\right) \cap \mathbf{B}^{2}} \rho d s \leq 2^{j+1} \int_{A_{j}} \rho d m_{2} \leq 2 \sqrt{\pi}\left(\int_{A_{j}} \rho^{2} d m_{2}\right)^{\frac{1}{2}} .
$$

Let us choose $j_{1} \in \mathbf{N}$ such that

$$
2 \sqrt{\pi}\left(\int_{A_{j_{1}}} \rho^{2} d m_{2}\right)^{\frac{1}{2}} \leq \frac{1}{2}
$$

and furthermore for every $k \in \mathbf{N}$ we choose $j_{k+1}>j_{k}$ such that

$$
2 \sqrt{\pi}\left(\int_{A_{j_{k+1}}} \rho^{2} d m_{2}\right)^{\frac{1}{2}} \leq \frac{1}{2^{k+1}} .
$$

Combining (3.3) with inequality (3.2) we get that

$$
\int_{S_{k}} \rho d s \leq \frac{1}{2^{k}} \quad \text { for every } k \in \mathbf{N}
$$

where $S_{k}:=S^{1}\left(\hat{\xi}, t_{j_{k}}\right) \cap \mathbf{B}^{2}$.

Let $x_{k}=\left(1-2^{-\left(j_{k}+1\right)}\right) \hat{\xi}$. Then $x_{k}$ is a point in the 'inner arc' of the 'semi'-ring $A_{j_{k}}$ for every $k \in \mathbf{N}$. Let us choose for every $k \in \mathbf{N}$ a curve $\gamma_{k}:[0,1] \rightarrow \mathbf{B}^{2}$ such that $\gamma_{k}(0)=0, \gamma_{k}(1)=x_{k}$ and

$$
\ell_{\rho}\left(\gamma_{k}\right) \leq d_{\rho}\left(0, x_{k}\right)+\frac{1}{2^{k}}
$$

Now it would be tempting to use curves $\gamma_{k}$ and apply the Arzela-Ascoli Theorem to define the curve $\gamma$, but in that case it may happen that $\gamma(1) \neq \hat{\xi}$. Hence we have to construct the curve $\gamma$ in a more clever way. In the following we concatenate the subcurves of curves $\gamma_{k}, k=1,2, \ldots$, joining $S_{k-1}$ and $S_{k}$ through the subarcs of $S_{k}$ to obtain the desired curve $\gamma$.

So, for every $k \in \mathbf{N}$ let $\alpha_{k}^{1}$ be the subcurve of $\gamma_{k}$ that joins 0 to the first point of $\gamma_{k} \cap S_{1}$. Furthermore, for every $i \in \mathbf{N}, 1 \leq i \leq k$, let $\alpha_{k}^{i}$ be the subcurve of $\gamma_{k}$ that joins the last point of $\gamma_{k} \cap S_{i-1}$ to the next point of $\gamma_{k} \cap S_{i}$. Then let $\beta_{k, l}^{i}$ be the subarc of $S_{i}$ that joins the points $\alpha_{k}^{i}(1)$ to $\alpha_{l}^{i+1}(0)$, where $k \geq i, l \geq i+1$. Then by inequality (3.4) we have that

$$
\ell_{\rho}\left(\beta_{k, l}^{i}\right) \leq \int_{S_{i}} \rho d s \leq \frac{1}{2^{i}} \quad \text { for every } k \geq i, l \geq i+1 .
$$

Next let us construct the concatenation $\gamma^{k}$ for every $k \in \mathbf{N}$,

$$
\gamma^{k}:=\alpha_{1}^{1} \star \beta_{1,2}^{1} \star \alpha_{2}^{2} \star \beta_{2,3}^{2} \star \ldots \star \alpha_{k}^{k} \star \beta_{k, k+1}^{k} .
$$

The curve $\gamma^{k}$ is well-defined. The path sequence $\left(\gamma^{k}\right)$ has a limit as $k \rightarrow \infty$ and we claim that this limit is the desired curve $\gamma$.

Firstly, $\lim _{t / 1}|\gamma(t)-\hat{\xi}|=0$ because, by construction, for every $j \in \mathbf{N}$ there is $t \in[0,1)$ such that $\left.\gamma\right|_{(t, 1)} \subset B\left(\hat{\xi}, 2^{-j}\right)$. 
Secondly, the curve $\gamma$ is $\rho$-rectifiable because the curve $\gamma^{k}$ is $\rho$-rectifiable for every $k \in \mathbf{N}$ with uniformly bounded length:

$$
\begin{aligned}
\ell_{\rho}\left(\gamma^{k}\right) & =\ell_{\rho}\left(\alpha_{1}^{1} \star \beta_{1,2}^{1} \star \alpha_{2}^{2} \star \beta_{2,3}^{2} \star \ldots \star \alpha_{k}^{k} \star \beta_{k, k+1}^{k}\right)=\sum_{i=1}^{k} \ell_{\rho}\left(\alpha_{i}^{i} \star \beta_{i, i+1}^{i}\right) \\
& \stackrel{(*)}{\leq} \ell_{\rho}\left(\alpha_{k}^{1} \star \bar{\beta}_{1, k}^{1} \star \beta_{1,2}^{1}\right)+\frac{1}{2}+\sum_{i=2}^{k}\left(\ell_{\rho}\left(\beta_{i, k}^{i-1} \star \alpha_{k}^{i} \star \bar{\beta}_{i, k}^{i} \star \beta_{i, i+1}^{i}\right)+\frac{1}{2^{i}}\right) \\
& \stackrel{(3.6)}{\leq} \ell_{\rho}\left(\gamma_{k}\right)+3 \sum_{i=1}^{k} \frac{1}{2^{i}}+\sum_{i=2}^{k} \frac{1}{2^{i-1}} \stackrel{(3.5)}{\leq} d_{\rho}\left(0, x_{k}\right)+4\left(1-\frac{1}{2^{k}}\right) \\
& \leq \operatorname{diam}_{\rho}\left(\mathbf{B}^{2}\right)+4<\infty
\end{aligned}
$$

and hence $\ell_{\rho}(\gamma)<\infty$. In the inequality $(*)$ we used the triangle inequality and inequality $(3.5)$.

Finally, because $\ell_{\rho}(\gamma)<\infty$, the $\operatorname{limit}_{t / 1} \gamma(t)$ exists in the sense of $d_{\rho}$, and we denote it by $\xi$. The limit $\xi$ must belong to the $\rho$-boundary $\partial_{\rho} \mathbf{B}^{n}$. Otherwise $\hat{\xi} \notin \mathbf{S}^{n-1}$.

When $n>2$, the proof is similar, but in inequality (3.6) we have to be more careful because the curve $\beta_{k, l}^{i}$ is now a curve in the $n$-1-dimensional sphere $S_{i}:=$ $S^{n-1}\left(\hat{\xi}, t_{j_{i}}\right) \cap \mathbf{B}^{n}$.

First, inequality (3.2) takes the form

$$
\int_{S^{n-1}\left(\hat{\xi}, t_{j}\right) \cap \mathbf{B}^{n}} \rho^{n} d m_{n-1} \leq 2^{j+1} \int_{A_{j}} \rho^{n} d m_{n} .
$$

For every $k \in \mathbf{N}$ we choose $j_{k} \in \mathbf{N}$ such that

$$
\int_{A_{j_{k}}} \rho^{n} d m_{n} \leq \frac{1}{2^{k}}
$$

and from inequality (3.7) we obtain

$$
\int_{S_{k}} \rho^{n} d m_{n-1} \leq \frac{2^{j_{k}+1}}{2^{k}} .
$$

Then, let for every $k, l \geq i, i, k, l \in \mathbf{N}$,

$$
\Gamma_{k, l}^{i}=\left\{\beta: \beta \text { joins points } \alpha_{k}^{i}(1) \text { and } \alpha_{l}^{i+1}(0) \text { on the surface } S_{i}\right\}
$$

and denote

$$
a_{k, l}^{i}=\inf _{\beta \in \Gamma_{k, l}^{i}} \ell_{\rho}(\beta)=\inf _{\beta \in \Gamma_{k, l}^{i}} \int_{\beta} \rho d s .
$$

If $a_{k, l}^{i}=0$ for some $i, k, l \in \mathbf{N}$, then there exists a curve $\beta_{k, l}^{i} \in \Gamma_{k, l}^{i}$ such that $\ell_{\rho}\left(\beta_{k, l}^{i}\right) \leq \frac{1}{2^{i}}$. If $a_{k, l}^{i}>0$, then

$$
\int_{\beta} \frac{\rho}{a_{k, l}^{i}} d s \geq 1 \quad \text { for every } \beta \in \Gamma_{k, l}^{i} .
$$

By (2.2) there is a constant $C=C(n)>0$ such that

$$
\frac{C}{t_{j_{i}}} \leq \bmod ^{S^{n-1}\left(\hat{\xi}, t_{j_{i}}\right)}\left(\Gamma_{k, l}^{i}\right) \leq \int_{S_{i}}\left(\frac{\rho}{a_{k, l}^{i}}\right)^{n} d m_{n-1} .
$$


By (3.8) we conclude that

$$
\left(a_{k, l}^{i}\right)^{n} \leq \frac{t_{j_{i}}}{C} \int_{S_{i}} \rho^{n} d m_{n-1} \leq \frac{2^{j_{i}+1}}{2^{j_{i}+i} C}=\frac{1}{2^{i-1} C} .
$$

Thus there exists a curve $\beta_{k, l}^{i} \in \Gamma_{k, l}^{i}$ such that

$$
\ell_{\rho}\left(\beta_{k, l}^{i}\right) \leq \frac{1}{2^{\frac{i-2}{n}} C^{\frac{1}{n}}} .
$$

Now we can apply the planar argument and the lemma is proven when we conclude that for every $k \in \mathbf{N}$,

$$
\ell_{\rho}\left(\gamma^{k}\right) \leq \operatorname{diam}_{\rho}\left(\mathbf{B}^{n}\right)+\frac{3 \cdot 2^{\frac{2}{n}}}{\left(2^{\frac{1}{n}}-1\right) C^{\frac{1}{n}}}+1<\infty .
$$

The last step is to prove uniqueness. Suppose that there exist two $\rho$-rectifiable curves, $\gamma$ and $\gamma^{\prime}$, with $\gamma(1)=\hat{\xi}=\gamma^{\prime}(1)$ in the Euclidean sense. When $n=2$, we deduce from inequality (3.4) that

$$
\lim _{t \nearrow 1} d_{\rho}\left(\gamma(t), \gamma^{\prime}(t)\right) \leq \lim _{k \rightarrow \infty} \int_{S_{k}} \rho d s \leq \lim _{k \rightarrow \infty} \frac{1}{2^{k}}=0 .
$$

When $n>2$, we obtain by the same argument as for inequality (3.9) that

$$
\lim _{t \nearrow 1} d_{\rho}\left(\gamma(t), \gamma^{\prime}(t)\right) \leq \lim _{k \rightarrow \infty} \frac{1}{2^{\frac{k-2}{n}} C^{\frac{1}{n}}}=0 .
$$

Thus the identification point $\xi$ in the $\rho$-boundary is uniquely determined by $\hat{\xi}$.

Remark 3.4. (i) In Lemma 3.3, instead of assuming $\rho \in L^{n}\left(\mathbf{B}^{n}\right)$ it would suffice to assume that for every $\hat{\xi} \in \mathbf{S}^{n-1}$ there exists $j_{\hat{\xi}} \in \mathbf{N}$ such that $\rho \in L^{p}\left(B\left(\hat{\xi}, 2^{-j_{\hat{\xi}}}\right) \cap\right.$ $\mathbf{B}^{n}$ ), where $p>n-1$ whenever $n>2$, and $p \geq 1$ when $n=2$. The idea of the proof is the same as in Lemma 3.3, but the proof is a bit more technical and we leave it to the reader. In this case the point $\hat{\xi}$ may determine more than one $\rho$-boundary point in $\partial_{\rho} \mathbf{B}^{n}$, as shown by simple examples.

(ii) If $\rho$ is a density which satisfies the volume growth condition VG for some function $\varphi$, and if $\operatorname{diam}_{\rho}\left(\mathbf{B}^{n}\right)<\infty$, then

$$
\int_{\mathbf{B}^{n}} \rho^{n} d m_{n} \leq \int_{B_{\rho}(0, M)} \rho^{n} d m_{n} \leq \varphi(M)<\infty
$$

for some $M>0$. Hence $\rho \in L^{n}\left(\mathbf{B}^{n}\right)$. Furthermore, if we could choose $\varphi(r)=B r^{n}$ for some $B>0$ (thus our density $\rho$ satisfies the condition $\operatorname{VG}(\mathrm{B})$ ), and if $\mathrm{HI}(\mathrm{A})$ held too, we would have the Gehring-Hayman theorem ([BKR], Theorem 3.1) to use. Consequently, we would have that $\ell_{\rho}([0, \hat{\xi}))<\infty$ for each radius $[0, \hat{\xi})$. This does not need to hold in the setting of Lemma 3.3. Indeed, fix $\hat{\xi} \in \mathbf{S}^{1}$ and define

$$
\rho(x)= \begin{cases}1, & \text { when } x \in \mathbf{B}^{2} \backslash B\left(\hat{\xi}, \frac{1}{4}\right) \text { or } \\ & x \in S^{1}\left(0,1-\frac{1}{2^{j}}\right) \cap B\left(\hat{\xi}, \frac{1}{4}\right), j=3,4, \ldots, \\ \frac{1}{|x-\hat{\xi}||\log | x-\hat{\xi}||}, & \text { "elsewhere" in } \mathbf{B}^{2},\end{cases}
$$


such that in each 'semi'-ring, $\left(B^{2}\left(0,1-\frac{1}{2^{j}}\right) \backslash \bar{B}^{2}\left(0,1-\frac{1}{2^{j-1}}\right)\right) \cap B\left(\hat{\xi}, \frac{1}{4}\right), j=3,4, \ldots$, $\rho$ grows very fast from 1 to $\frac{1}{|x-\hat{\xi}||\log | x-\hat{\xi}||}$ so that $\rho$ is continuous and that 'most' of the time $\rho(x)=\frac{1}{|x-\hat{\xi}||\log | x-\hat{\xi}||}$. Then $\operatorname{diam}_{\rho}\left(\mathbf{B}^{2}\right)<\infty$ and

$$
\begin{aligned}
\mu_{\rho}\left(\mathbf{B}^{2}\right) & =\int_{\mathbf{B}^{2}} \rho^{2} d m_{2} \leq \int_{\mathbf{B}^{2} \backslash B(\hat{\xi}, 1 / 4)} d x+\int_{B(\hat{\xi}, 1 / 4)} \frac{1}{|x-\hat{\xi}|^{2}|\log | x-\left.\hat{\xi}\right|^{2}} d x \\
& \leq C_{1}+\int_{0}^{2 \pi} \int_{0}^{1 / 4} \frac{t}{t^{2}|\log t|^{2}} d t=C_{2}<\infty,
\end{aligned}
$$

but

$$
\ell_{\rho}\left(\left[0, \hat{\xi}[) \approx \frac{3}{4}+\int_{3 / 4}^{1} \frac{1}{(1-t)|\log (1-t)|} d t=\infty .\right.\right.
$$

When we combine Lemma 3.3 with Lemma 3.2, we obtain the following conclusion.

Corollary 3.5. Let $\rho$ satisfy $\log -V G$. If $\operatorname{diam}_{\rho}\left(\mathbf{B}^{n}\right)<\infty$, then the homeomorphic identity map id: $\left(\mathbf{B}^{n}, d_{\rho}\right) \rightarrow\left(\mathbf{B}^{n},|\cdot|\right)$ has a continuous and bijective extension $i:\left(\mathbf{B}^{n} \cup \partial_{\rho} \mathbf{B}^{n}, d_{\rho}\right) \rightarrow\left(\mathbf{B}^{n} \cup \mathbf{S}^{n-1},|\cdot|\right)$.

Proof. From Lemma 3.2 we know that we can identify every $\xi \in \partial_{\rho} \mathbf{B}^{n}$ uniquely with some point $\hat{\xi} \in \mathbf{S}^{n-1}$. The identity map $i$ is well-defined, and by the same argument used for inequalities (RHS) and (LHS) in Lemma 3.2 it follows that this map is also continuous. From Lemma 3.3 we deduce that $i$ is surjective and injective because $\rho \in L^{n}\left(\mathbf{B}^{n}\right)$.

If we assume that the density $\rho$ satisfies the Harnack type inequality $\mathrm{HI}(\mathrm{A})$, we can relax the logarithmic volume growth condition log-VG, and the previous result still holds.

Theorem 3.6. Let $\rho$ satisfy $H I(A)$ and $V G$, where $\varphi(r)=B r^{n-\epsilon}$ for some $0<\epsilon<\frac{1}{1+\log A}$ and for some $B>0$. If $\operatorname{diam}_{\rho}\left(\mathbf{B}^{n}\right)<\infty$, then the homeomorphic identity map id: $\left(\mathbf{B}^{n}, d_{\rho}\right) \rightarrow\left(\mathbf{B}^{n},|\cdot|\right)$ has a continuous and bijective extension $i:\left(\mathbf{B}^{n} \cup \partial_{\rho} \mathbf{B}^{n}, d_{\rho}\right) \rightarrow\left(\mathbf{B}^{n} \cup \mathbf{S}^{n-1},|\cdot|\right)$.

Proof. The hard part is to prove that the map $i$ is well-defined. Let $\xi \in \partial_{\rho} \mathbf{B}^{n}$ and let $\left(x_{k}\right) \subset \mathbf{B}^{n}$ be a $d_{\rho^{-}}$Cauchy sequence which converges to the point $\xi$. Because $\mathbf{B}^{n} \cup \mathbf{S}^{n-1}$ is compact, switching to a subsequence, we may assume that $\left(x_{k}\right)$ converges in the Euclidean sense to some point in $\mathbf{S}^{n-1}$. Let this limit point be $\hat{\xi}$.

Let $\left(y_{k}\right) \subset \mathbf{B}^{n}$ be another $d_{\rho}$-Cauchy sequence that converges to $\xi$ and let us assume that $\left(y_{k}\right)$ converges in the Euclidean sense to a point $\hat{\eta} \neq \hat{\xi}$. Let $\delta>0$ be so small that $0 \notin B_{\rho}(\xi, 14 \delta)$, and let $N \in \mathbf{N}$ be so large that when $k>N$, $x_{k}, y_{k} \in B_{\rho}(\xi, \delta), x_{k} \in B(\hat{\xi},|\hat{\xi}-\hat{\eta}| / 10)$ and $y_{k} \in B(\hat{\eta},|\hat{\xi}-\hat{\eta}| / 10)$. Let $k>N$ and let $\gamma_{k} \subset \mathbf{B}^{n}$ be a curve which joins points $x_{k}$ and $y_{k}$ so that $\ell_{\rho}\left(\gamma_{k}\right) \leq 2 d_{\rho}\left(x_{k}, y_{k}\right)$. Then $\gamma_{k} \subset B_{\rho}(\xi, 2 \delta)$ and $\ell\left(\gamma_{k}\right) \geq\left|x_{k}-y_{k}\right| \geq \frac{4}{5}|\hat{\xi}-\hat{\eta}|$.

Denote $\ell_{\rho}\left(\gamma_{k}\right)=r$. Let $x \in \gamma_{k}$, and let us consider the $\rho$-ball $B_{\rho}(x, 3 r)$. Let $E=\left\{\omega \in \mathbf{S}^{n-1}:[0, \omega) \cap \gamma_{k} \neq \emptyset\right\}$, where $[0, \omega)$ is the radius that joins 0 and $\omega$. Let $E_{1}=\left\{\omega \in E: d_{\rho}(z, \omega) \leq r\right.$ for some $\left.z \in \gamma_{k}\right\}$ and $E_{2}=E \backslash E_{1}=\left\{\omega \in E: d_{\rho}(z, \omega)>\right.$ $r$ for every $\left.z \in \gamma_{k}\right\}$. 
We give the rest of the proof in detail when $n=2$ and give the idea when $n>2$. Thus, let $n=2$. Because the 1-dimensional Lebesgue measure of $E$ is $m_{1}(E) \geq$ $\frac{4}{5}|\hat{\xi}-\hat{\eta}|$, it follows that $m_{1}\left(E_{i}\right) \geq \frac{2}{5}|\hat{\xi}-\hat{\eta}|$ for $i=1$ or $i=2$.

Suppose $m_{1}\left(E_{1}\right) \geq \frac{2}{5}|\hat{\xi}-\hat{\eta}|$. Only in a null set $\hat{E} \subset E_{1}$ it is possible that $\ell_{\rho}([t \omega, \omega))=\infty$, where $\omega \in \hat{E}$, and $0<t<1$ is such that $[t \omega, \omega) \subset B_{\rho}(x, 3 r)$. Otherwise, if $m_{1}(\hat{E})>0$, it would hold that

$$
\mu_{\rho}\left(B_{\rho}(x, 3 r)\right)=\int_{B_{\rho}(x, 3 r)} \rho^{2} d m_{2} \geq \int_{\hat{E}} \int_{t}^{1} \rho^{2}(s \omega) d s d \omega=\infty .
$$

This is a contradiction because of the volume growth condition VG.

Let $\omega \in E_{1} \backslash \hat{E}$ and let $\left.t_{\omega} \in\right] 0,1\left[\right.$ be such that $\ell_{\rho}\left(\left[t_{\omega} \omega, \omega\right)\right)=r$. Because of the choice of $\delta$ and the previous discussion, such a $t_{\omega}$ exists. Now $\left[t_{\omega} \omega, \omega\right) \subset B_{\rho}(x, 3 r)$ and from Hölder's inequality we obtain

$$
r=\int_{\left[t_{\omega} \omega, \omega\right)} \rho d s=\int_{t_{\omega}}^{1} \rho(t \omega) d t \leq\left(\int_{t \omega}^{1} \rho^{2}(t \omega) d t\right)^{\frac{1}{2}}\left(1-t_{\omega}\right)^{\frac{1}{2}} .
$$

Furthermore, using this we deduce that

$$
\begin{aligned}
\mu_{\rho}\left(B_{\rho}(x, 3 r)\right) & =\int_{B_{\rho}(x, 3 r)} \rho^{2} d m_{2} \geq \int_{E_{1} \backslash \hat{E}} \int_{t_{\omega}}^{1} \rho^{2}(t \omega) d t d \omega \\
& \geq \int_{E_{1} \backslash \hat{E}} \frac{r^{2}}{1-t_{\omega}} d \omega \geq \frac{2 r^{2}}{5 \max _{\omega \in E_{1} \backslash \hat{E}}\left(1-t_{\omega}\right)}|\hat{\xi}-\hat{\eta}| .
\end{aligned}
$$

Because one can cover every line segment $[0, y] \subset \mathbf{B}^{2}$ with no more than $1+\log \frac{1}{1-|y|}$ balls $B_{z}=B\left(z, \frac{1}{2}(1-|z|)\right), z \in \mathbf{B}^{2}$, by $\mathrm{HI}(\mathrm{A})$, we have that for every $\omega \in \mathbf{S}^{1}$ and for every $0<t<1$

$$
\rho(0) \leq A^{1+\log \frac{1}{1-t}} \rho(t \omega)=A\left(\frac{1}{1-t}\right)^{\log A} \rho(t \omega)
$$

Thus for each $w \in E_{1} \backslash \hat{E}$ we obtain

$$
r=\int_{t_{\omega}}^{1} \rho(t \omega) d t \geq \int_{t_{\omega}}^{1} \frac{\rho(0)}{A}(1-t)^{\log A} d t=\frac{\rho(0)}{A(1+\log A)}\left(1-t_{\omega}\right)^{1+\log A},
$$

and combining this with (3.10) we get

$$
\mu_{\rho}\left(B_{\rho}(x, 3 r)\right) \geq \frac{2}{5}\left(\frac{\rho(0)}{A(1+\log A)}\right)^{\frac{1}{1+\log A}}|\hat{\xi}-\hat{\eta}| r^{2-\frac{1}{1+\log A}} .
$$

For any choice of $0<\epsilon<\frac{1}{1+\log A}$ the volume growth condition VG will not hold for each $r>0$.

Suppose $m_{1}\left(E_{2}\right) \geq \frac{2}{5}|\hat{\xi}-\hat{\eta}|$. Let $\omega \in E_{2}$. Let $t_{\omega}, t_{\omega}^{\prime} \in(0,1)$ be such that $t_{\omega}<t_{\omega}^{\prime}$, $t_{\omega} \omega \in \gamma_{k},\left[t_{\omega} \omega, t_{\omega}^{\prime} \omega\right] \subset[0, \omega) \cap B_{\rho}(x, 3 r)$ and $\ell_{\rho}\left(\left[t_{\omega} \omega, t_{\omega}^{\prime} \omega\right]\right)=r$. Now as in the previous case we have

$$
r \leq\left(\int_{t_{\omega}}^{t_{\omega}^{\prime}} \rho^{2}(t \omega) d t\right)^{\frac{1}{2}}\left(t_{\omega}^{\prime}-t_{\omega}\right)^{\frac{1}{2}}
$$

and moreover

$$
\mu_{\rho}\left(B_{\rho}(x, 3 r)\right) \geq \frac{2 r^{2}}{5 \max _{\omega \in E_{2}}\left(t_{\omega}^{\prime}-t_{\omega}\right)}|\hat{\xi}-\hat{\eta}| \geq \frac{2 r^{2}}{5 \max _{\omega \in E_{2}}\left(1-t_{\omega}\right)}|\hat{\xi}-\hat{\eta}| .
$$


Because of the choices at the beginning of the proof, the curve $\gamma_{k}$ is not a subset of $B(y, 1 / 2(1-|y|))$ for any $y \in \gamma_{k}$, and this yields with $\mathrm{HI}(\mathrm{A})$ that for each $\omega \in E_{2}$

$$
r=\int_{\gamma_{k}} \rho d s \geq \frac{1}{A} \rho\left(t_{\omega} \omega\right) \ell\left(\gamma_{k} \cap B_{t_{\omega} \omega}\right) \geq \frac{1}{2 A} \rho\left(t_{\omega} \omega\right)\left(1-t_{\omega}\right) .
$$

Hence this with (3.11) gives us

$$
r \geq \frac{1}{2 A} \max _{\omega \in E_{2}}\left(\rho\left(t_{\omega} \omega\right)\left(1-t_{\omega}\right)\right) \geq \frac{\rho(0)}{2 A^{2}} \max _{\omega \in E_{2}}\left(1-t_{\omega}\right)^{1+\log A} .
$$

Furthermore, combining this with (3.12) we obtain

$$
\mu_{\rho}(B(x, 3 r)) \geq \frac{2}{5}\left(\frac{\rho(0)}{2 A^{2}}\right)^{\frac{1}{1+\log A}}|\hat{\xi}-\hat{\eta}| r^{2-\frac{1}{1+\log A}} .
$$

Again, for any choice of $0<\epsilon<\frac{1}{1+\log A}$ the volume growth condition VG will not hold for each $r>0$. Hence the identity map $i$ is well-defined.

When $n>2$, the proof is more technical, because the set $E$ may have zero $n$-1-dimensional Lebesgue measure. We use here the same technique as in [KL, Theorem 1.1]. Let $\mathscr{W}$ be a Whitney decomposition of $\mathbf{B}^{n}$ - that is, a collection of dyadic cubes $Q \subset \mathbf{B}^{n}$ having pairwise disjoint interiors and satisfying the condition

$$
\operatorname{diam}(Q) \leq d\left(Q, \mathbf{S}^{n-1}\right) \leq 4 \operatorname{diam}(Q)
$$

(see [St] for the existence and properties of Whitney decompositions). Cubes that intersect the line segment $\left[t_{\omega} \omega, \omega\right), \omega \in E_{1}$, or the line segment $\left[t_{\omega} \omega, t_{\omega}^{\prime} \omega\right], \omega \in E_{2}$, are included in the $\rho$-ball $B_{\rho}\left(x,\left(3+2 A^{3}\right) r\right)$. Hence we can estimate the $\mu_{\rho}$-measure of $B_{\rho}\left(x,\left(3+2 A^{3}\right)\right)$ from below with the help of these intersecting cubes. Because the Hausdorff 1-content of $E$ is $\mathscr{H}_{\infty}^{1}(E) \geq \frac{\operatorname{diam}\left(\gamma_{k}\right)}{2} \geq \frac{2}{5}|\hat{\xi}-\hat{\eta}|$, using Frostman's Lemma ([Ma], [KL]) we obtain that

$$
\mu_{\rho}\left(B_{\rho}\left(x,\left(3+2 A^{3}\right) r\right)\right) \geq C|\hat{\xi}-\hat{\eta}| r^{n-\frac{1}{1+\log A}},
$$

where $C=C(A, n)>0$ is a constant. As in the case $n=2$, the same choice of $\epsilon$ leads us to a contradiction.

The proof above also tells us that the map $i$ is continuous. From Lemma 3.3 we obtain that it is both surjective and injective.

For the rest of the paper we combine these two conditions of the density $\rho$, Corollary 3.5 and Theorem 3.6, and we consider densities $\rho$ whose associated measure $\mu_{\rho}$ has allowable growth.

What about the continuity of the inverse map $\left(\left.i\right|_{\partial_{\rho} \mathbf{B}^{n}}\right)^{-1}:\left(\mathbf{S}^{n-1},|\cdot|\right) \rightarrow\left(\partial_{\rho} \mathbf{B}^{n}, d_{\rho}\right)$ ? Here is an example where it is not continuous:

Example 3.7. As pictured in Figure 1, we consider the simply connected plane domain

$$
\Omega=(0,1) \times(0,1) \backslash \bigcup_{k=1}^{\infty}\left(\left\{\frac{1}{2^{k}}\right\} \times\left[0, \frac{1}{2}\right]\right)
$$

equipped with its internal metric $d_{I}$. Let $\rho(x):=\left|f^{\prime}(x)\right|$, where $f:\left(\mathbf{B}^{2},|\cdot|\right) \rightarrow(\Omega,|\cdot|)$ is a conformal map. Then for each pair of points $x, y \in \mathbf{B}^{2}$,

$$
d_{\rho}(x, y)=d_{I}(f(x), f(y)),
$$

as we saw in the introduction. Thus $\left(\mathbf{B}^{2}, d_{\rho}\right)$ is bounded. 


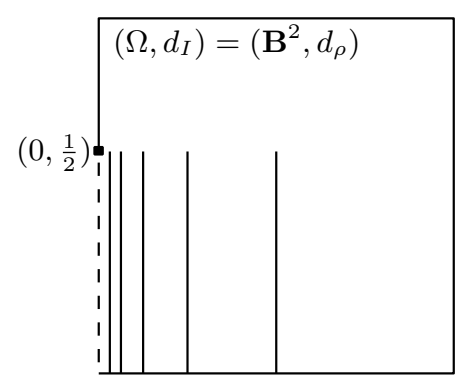
$\mathbf{S}^{1}$.

Figure 1. Example of a domain $\left(\Omega, d_{I}\right)=\left(\mathbf{B}^{2}, d_{\rho}\right)$, whose $\rho$-boundary is not homeomorphic to

The density $\rho$ satisfies the volume growth condition $\operatorname{VG}(\pi)$. Hence $\left(\mathbf{B}^{2}, d_{\rho}\right)$ satisfies the assumptions of Corollary 3.5 (or even the assumptions of Theorem 3.6). The boundary $\partial_{\rho} \mathbf{B}^{2}=\partial_{\mathrm{I}} \Omega$ is closed and bounded but not compact. Indeed, let $\left(x_{k}\right) \subset \partial_{\mathrm{I}} \Omega$ be the sequence of the "midpoints",

$$
x_{k}=\left(\frac{3}{2^{k+2}}, 0\right) \text {. }
$$

This sequence cannot have a convergent subsequence because

$$
d_{I}\left(x_{k}, x_{k+1}\right) \geq 1
$$

for every $k \in \mathbf{N}$. Hence the identity map $\left.i\right|_{\partial_{\rho} \mathbf{B}^{n}}$ is not a homeomorphism.

We need to assume something about the $\rho$-boundary $\partial_{\rho} \mathbf{B}^{n}$ to obtain a homeomorphic identity map. In fact, it is enough to assume that $\partial_{\rho} \mathbf{B}^{n}$ is compact, as the next section shows.

\section{Proof of the main theorem}

Now we are able to prove the equivalences (i) $\Leftrightarrow$ (ii) $\Leftrightarrow$ (iii) of our main theorem, Theorem 1.1.

Proof of Theorem 1.1. The implications (iii) $\Rightarrow$ (ii) and (ii) $\Rightarrow$ (i) are clear, because $\left(\mathbf{B}^{n} \cup \mathbf{S}^{n-1},|\cdot|\right)$ is compact and $\partial_{\rho} \mathbf{B}^{n}=\overline{\mathbf{B}}_{\rho}^{n} \backslash \mathbf{B}_{\rho}^{n}$ is closed. The implication (ii) $\Rightarrow$ (iii) follows from Corollary 3.5 and Theorem 3.6 through a well-known fact that a continuous bijection from a compact space to a Hausdorff space is a homeomorphism (see for example $[\mathrm{Mu}]$ ).

Let us prove (i) $\Rightarrow$ (ii). Assume that $\partial_{\rho} \mathbf{B}^{n}$ is compact. Let $\left\{A_{\alpha}\right\}_{\alpha \in I}$ be a $d_{\rho}$-open cover of the set $\mathbf{B}^{n} \cup \partial_{\rho} \mathbf{B}^{n}$. Let $J \subset I$ be a maximal index set such that for each $\alpha \in J$ it holds that $A_{\alpha} \cap \partial_{\rho} \mathbf{B}^{n} \neq \emptyset$. Because $\partial_{\rho} \mathbf{B}^{n}$ is compact, there exists a finite index set $J^{\prime} \subset J$ such that

$$
\partial_{\rho} \mathbf{B}^{n} \subset \bigcup_{\alpha \in J^{\prime}} A_{\alpha}
$$

Now $W:=\left(\mathbf{B}^{n} \cup \partial_{\rho} \mathbf{B}^{n}\right) \backslash \bigcup_{\alpha \in J^{\prime}} A_{\alpha}$ is $d_{\rho^{-}}$-closed and $W \subset \mathbf{B}^{n}$. Because id: $\mathbf{B}_{\rho}^{n} \rightarrow \mathbf{B}^{n}$ is always a homeomorphism, $W$ is closed also in the Euclidean sense and thus $W$ is compact. Consequently, $W$ is compact in the $\rho$-metric. Moreover, there is a finite index set $I^{\prime} \subset I$ such that

$$
W \subset \bigcup_{\alpha \in I^{\prime}} A_{\alpha}
$$


and hence we find a finite subcover $I^{\prime} \cup J^{\prime}$ such that

$$
\mathbf{B}^{n} \cup \partial_{\rho} \mathbf{B}^{n} \subset \bigcup_{\alpha \in I^{\prime} \cup J^{\prime}} A_{\alpha}
$$

as desired.

In the planar case there is another equivalent statement as given in Theorem 1.1 (iv). For this, recall that a set $A$ is locally connected at a point $a \in A$, if for every neighborhood $U \subset A$ of $a$ there exists a connected neighborhood $V \subset U$ of $a$. A set $A$ is locally connected, if it is locally connected at every point $a \in A$. Notice also that $\partial_{\mathrm{I}} \Omega$ in the example 3.7 is not locally connected at $\left(0, \frac{1}{2}\right)$.

Proof of Theorem 1.1. (iii) $\Leftrightarrow$ (iv). If the identity map $i:\left(\mathbf{B}^{2} \cup \partial_{\rho} \mathbf{B}^{2}, d_{\rho}\right) \rightarrow$ $\left(\mathbf{B}^{2} \cup \mathbf{S}^{1},|\cdot|\right)$ is a homeomorphism, then $\partial_{\rho} \mathbf{B}^{2}$ is locally connected because a homeomorphism preserves this property.

We assume that $\partial_{\rho} \mathbf{B}^{2}$ is locally connected and $\operatorname{diam}_{\rho}\left(\mathbf{B}^{2}\right)<\infty$. From Corollary 3.5 and Theorem 3.6 it follows that the identity map $i:\left(\mathbf{B}^{2} \cup \partial_{\rho} \mathbf{B}^{2}, d_{\rho}\right) \rightarrow$ $\left(\mathbf{B}^{2} \cup \mathbf{S}^{1},|\cdot|\right)$ is a continuous bijection. Thus it is enough to show that $i^{-1}$ is continuous. For that, let $\hat{\xi} \in \mathbf{S}^{1}$ and let $\xi \in \partial_{\rho} \mathbf{B}^{2}$ be the identification point of $\hat{\xi}$. Let $\epsilon>0$ and let us first search for $\delta>0$ such that $i^{-1}\left(B(\hat{\xi}, \delta) \cap \mathbf{S}^{1}\right) \subset B_{\rho}(\xi, \epsilon) \cap \partial_{\rho} \mathbf{B}^{2}$.

The ball $B_{\rho}(\xi, \epsilon) \cap \partial_{\rho} \mathbf{B}^{2}$ contains a connected neighborhood $U$ of $\xi$ and thus $i(U)=\hat{U} \subset \mathbf{S}^{1}$ is a connected subarc of $\mathbf{S}^{1}$. Let us prove that $\hat{\xi}$ is an interior point of $\hat{U}$. In other words, we prove that $\hat{\xi}$ is not an endpoint of $\hat{U}$.

Let $r=d_{\rho}\left(\xi, \partial_{\rho} \mathbf{B}^{2} \backslash U\right)>0$ and let $\gamma:[0,1) \rightarrow \mathbf{B}^{n}$ be the $\rho$-rectifiable curve constructed in Lemma 3.3 with $\gamma(1)=\xi$. Let $t_{0} \in[0,1)$ be such that $\left.\gamma\right|_{\left(t_{0}, 1\right)} \subset$ $B_{\rho}\left(\xi, \frac{r}{2}\right) \subset \mathbf{B}^{2} \cup \partial_{\rho} \mathbf{B}^{2}$. If $\hat{\xi}$ were an endpoint of $\hat{U}$, then there would exist a nondegenerate connected set $E \subset \mathbf{S}^{1} \backslash \hat{U}$ such that $\hat{\xi} \in \bar{E}$ and therefore

$$
\bmod \left(\left.\gamma\right|_{\left(t_{0}, 1\right)}, E ; \mathbf{B}^{2}\right)=\infty .
$$

On the other hand the $\rho$-length of any curve $\alpha$ that connects $\left.\gamma\right|_{\left(t_{0}, 1\right)}$ and $i^{-1}(E)$ is at least $\frac{r}{2}$. Hence, when we choose $\tilde{\rho}=\frac{2}{r} \rho$, we have that $\ell_{\tilde{\rho}}(\alpha) \geq 1$. Because $\mu_{\rho}$ has allowable growth and $\mathbf{B}_{\rho}^{2}$ is bounded, we conclude that $\rho \in L^{2}\left(\mathbf{B}^{2}\right)$ and therefore

$$
\bmod \left(\left.\gamma\right|_{\left(t_{0}, 1\right)}, E ; \mathbf{B}^{2}\right) \leq \int_{\mathbf{B}^{2}} \tilde{\rho}^{2} d m_{2}=\frac{4}{r^{2}} \int_{\mathbf{B}^{2}} \rho^{2} d m_{2}<\infty
$$

which is a contradiction. Thus $\hat{\xi} \in \hat{U}$ is an interior point and there exists $\delta=$ $\delta(\epsilon, \hat{\xi})>0$ such that $B(\hat{\xi}, \delta) \cap \mathbf{S}^{1} \subset \hat{U}$. Thus $\left(\left.i\right|_{\partial_{\rho} \mathbf{B}^{2}}\right)^{-1}: \mathbf{S}^{1} \rightarrow \partial_{\rho} \mathbf{B}^{2}$ is continuous. Furthermore, $\partial_{\rho} \mathbf{B}^{2}$ is compact, and so from Theorem 1.1 (i) $\Leftrightarrow$ (iii) we deduce that $i:\left(\mathbf{B}^{2} \cup \partial_{\rho} \mathbf{B}^{2}, d_{\rho}\right) \rightarrow\left(\mathbf{B}^{2} \cup \mathbf{S}^{1},|\cdot|\right)$ is a homeomorphism.

The assumption of the boundedness of $\left(\mathbf{B}^{2}, d_{\rho}\right)$ is crucial. Indeed, let $H^{+}=$ $\left\{x=\left(x_{1}, x_{2}\right) \in \mathbf{R}^{2}: x_{2}>0\right\}$ and $f: \mathbf{B}^{2} \rightarrow H^{+}$be a conformal mapping. Then $\partial_{\rho} \mathbf{B}^{2}=\partial H^{+}=\mathbf{R} \times\{0\}$ which is locally connected, but the identity map $i$ is not even bijective. Here our density is again $\rho(x)=\left|f^{\prime}(x)\right|$.

We close this paper by showing that Theorem 1.1 (iii) $\Leftrightarrow$ (iv) holds only in dimension two. For simplicity we give an example in dimension three; it can easily be extended to higher dimensions. 
Example 4.1. There exists a domain $\Omega \subset \mathbf{R}^{3}$ such that equipping it with its internal metric $d_{I}$ we obtain that $\Omega_{I}$ corresponds to $\mathbf{B}_{\rho}^{3}$ for the conformal density $\rho$, and the boundary $\partial_{\rho} \mathbf{B}^{3}$ is locally connected but not compact. In fact, $\mathbf{B}_{\rho}^{3}$ is also locally connected at each $\xi \in \partial_{\rho} \mathbf{B}^{3}$.

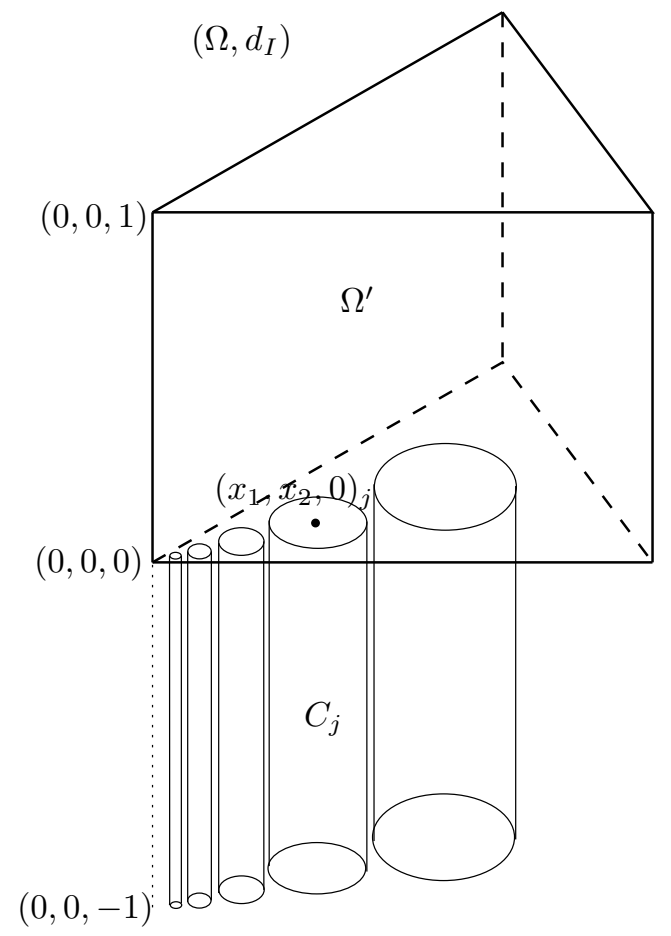

Figure 2. Example of a domain $\Omega_{I}$, whose boundary $\partial_{\mathrm{I}} \Omega$ is locally connected but not compact.

As pictured in Figure 2, let $\Omega \subset \mathbf{R}^{3}$ be the domain that is the union of an open triangular prism $\Omega^{\prime}$ and open cylinders $\bigcup_{j=1}^{\infty} C_{j}$. The length of each cylinder $C_{j}$ is one and the sequence $\left(C_{j}\right)_{1}^{\infty}$ converges with respect to the Euclidean Hausdorff distance to the line segment $\{0\} \times\{0\} \times(-1,0)$. The boundary $\partial_{\mathrm{I}} \Omega$ is locally connected and $\Omega$ is locally connected along $\partial_{\mathrm{I}} \Omega-$ also at the origin - but $\partial_{\mathrm{I}} \Omega$ is not compact. Thus $\bar{\Omega}_{I}$ is not homeomorphic to $\overline{\mathbf{B}}^{3}$. For example, if $x_{j}$ is a point chosen from the bottom of $C_{j}$, then the sequence $\left(x_{j}\right)_{1}^{\infty}$ does not have a convergent subsequence because $d_{I}\left(x_{j}, x_{j+1}\right) \geq 2$ for every $j \in \mathbf{N}$.

Let us assume that there is a $K$-quasiconformal mapping $f: \mathbf{B}^{3} \rightarrow \Omega$. Setting

$$
\rho(x)=\frac{\operatorname{dist}(f(x), \partial \Omega)}{1-|x|}
$$

we obtain a density that satisfies the assumptions of Theorem 1.1. In fact, $\rho$ even satisfies the conditions $\mathrm{HI}(\mathrm{A})$ and $\mathrm{VG}(\mathrm{B})$ (cf. [BKR, §2.4]). Moreover there exists a constant $C=C(K) \geq 1$ such that

$$
\frac{1}{C} d_{I}(f(x), f(y)) \leq d_{\rho}(x, y) \leq C d_{I}(f(x), f(y)) .
$$

It follows that $\partial_{\rho} \mathbf{B}^{3}$ is locally connected and $\mathbf{B}_{\rho}^{3}$ is locally connected at each $\xi \in$ $\partial_{\rho} \mathbf{B}^{3}$.

It remains to verify that there really is such a map $f$. There exists a quasiconformal mapping $f_{1}$ that maps the unit ball $\mathbf{B}^{3}$ onto the triangular prism $\Omega^{\prime}$. Then there 
is a bi-Lipschitz map $g_{1}$ which maps a half ball

$$
B_{+}^{3}(y, r)=\left\{x=\left(x_{1}, x_{2}, x_{3}\right) \in \mathbf{R}^{3}:|x-y|<r, x_{3}-y_{3}>0\right\}
$$

to a half ball with a small dent $B_{+}^{3}(y, r) \backslash \overline{B_{+}^{3}}(y, t r), r>0,0<t<1$. The biLipschitz constant depends only on $0<t<1$, and thus it is same for every $y \in \mathbf{R}^{3}$ and every $r>0$. We can map the set $B_{+}^{3}(y, r) \backslash \overline{B_{+}^{3}}(y, t r)$ quasiconformally by $g_{2}$ to a cylinder $B^{2}(y, r) \times\left(y_{3}, y_{3}-1\right)(\mathrm{cf}$. $[\mathrm{GV}])$. Here the quasiconformality constant depends only on $0<t<1$. We further have a bi-Lipschitz map $g_{3}$ which maps the cylinder with a dent

$$
\left(B^{2}(y, r) \times\left(y_{3}, y_{3}+2 r\right)\right) \backslash B_{+}^{3}(y, r)
$$

to the cylinder $B^{2}(y, r) \times\left(y_{3}, y_{3}+2 r\right)$ so that the boundary values of $g_{3}$ coincide with those of $g_{2}$ on the boundary of $B_{+}^{3}(y, r) \backslash \overline{B_{+}^{3}}(y, t r)$. Figure 3 clarifies how maps $g_{j}, j=1,2,3$, work.
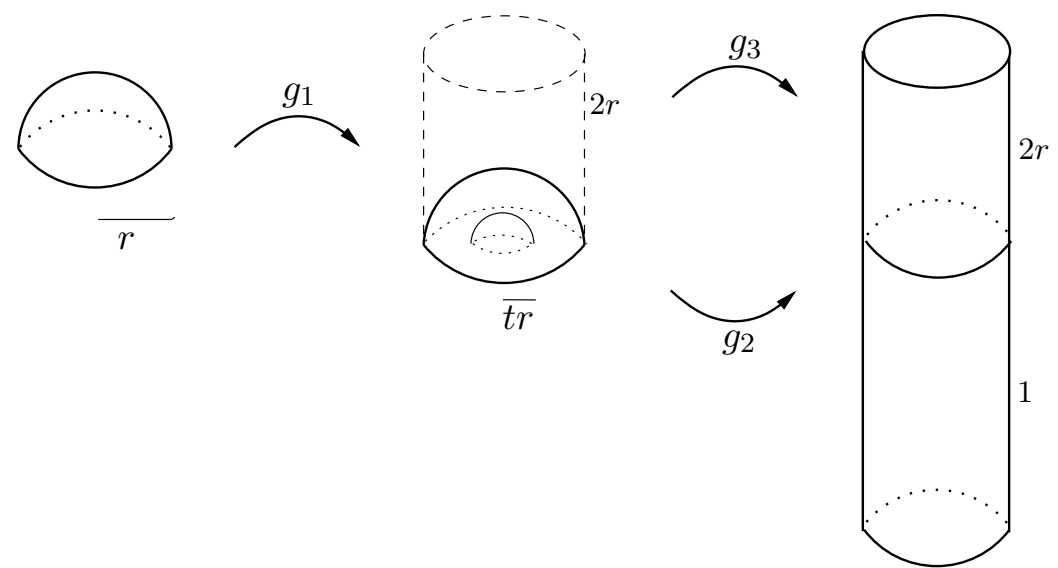

Figure 3. How to map a half ball to a cylinder quasiconformally?

Thus let $\bigcup_{j=1}^{\infty} B_{j}^{3} \subset \Omega^{\prime}$ be the collection of half balls, $B_{j}^{3}=B_{+}^{3}\left(\left(x_{1}, x_{2}, 0\right)_{j}, r_{j}\right)$, in the bottom of the triangular prism so that the half ball $B_{j}^{3}$ is above the desired cylinder $C_{j}$ and the radius $r_{j}$ is the same as the radius of $C_{j}$. Fix $0<t<1$. Now we define a map, $f_{2}: \Omega^{\prime} \rightarrow \Omega^{\prime} \backslash \bigcup_{j=1}^{\infty} \overline{B_{+}^{3}}\left(\left(x_{1}, x_{2}, 0\right)_{j}, t r_{j}\right)$ by setting

$$
f_{2}(x)= \begin{cases}x, & \text { when } x \in \Omega^{\prime} \backslash\left(\bigcup_{j=1}^{\infty} B_{j}^{3}\right), \\ g_{1}(x), & \text { when } x \in \bigcup_{j=1}^{\infty} B_{j}^{3} .\end{cases}
$$

Our map $f_{2}$ is clearly quasiconformal; the constants above depend only on $t$, which is fixed. Then we define, $f_{3}: \Omega^{\prime} \backslash \bigcup_{j=1}^{\infty} \overline{B_{+}^{3}}\left(\left(x_{1}, x_{2}, 0\right)_{j}, t r_{j}\right) \rightarrow \Omega$,

$$
f_{3}(x)= \begin{cases}x, & \text { when } x \in \Omega^{\prime} \backslash \bigcup_{j=1}^{\infty} B^{2}\left(\left(x_{1}, x_{2}, 0\right)_{j}, r_{j}\right) \times\left(0,2 r_{j}\right), \\ g_{3}(x), & \text { when } x \in \bigcup_{j=1}^{\infty}\left(B^{2}\left(\left(x_{1}, x_{2}, 0\right)_{j}, r_{j}\right) \times\left(0,2 r_{j}\right)\right) \backslash \overline{B_{j}^{3}}, \\ g_{2}(x), & \text { when } x \in \bigcup_{j=1}^{\infty}\left(B_{j}^{3} \backslash \overline{B_{+}^{3}}\left(\left(x_{1}, x_{2}, 0\right)_{j}, t r_{j}\right)\right) .\end{cases}
$$

This is also a quasiconformal mapping when we glue maps $g_{2}$ and $g_{3}$ naturally together. Then the quasiconformal mapping $f=f_{3} \circ f_{2} \circ f_{1}$ maps the unit ball $\mathbf{B}^{3}$ onto $\Omega$. 
Acknowledgement. The author is greatly obliged to Pekka Koskela for his patient guidance and myriad helpful suggestions. The author is also deeply indebted to referees for their valuable comments and reading the manuscript so carefully.

\section{References}

[BHR] Bonk, M., J. Heinonen, and S. Rohde: Doubling conformal densities. - J. Reine Angew. Math. 541, 2001, 117-141.

[BK] Bonk, M., and P. Koskela: Conformal densities and size of the boundary. - Amer. J. Math. 124, 2002, 1247-1287.

[BKR] Bonk, M., P. Koskela, and S. Rohde: Conformal metrics on the unit ball in Euclidean space. - Proc. London Math. Soc. 270:3, 1998, 635-664.

[Ge] Gehring, F. W.: Symmetrization of rings in space. - Trans. Amer. Math. Soc. 101:3, 1961, 499-519.

[GV] Gehring, F. W., and J. VÄISÄLÄ: The coefficients of quasiconformality of domains in space. - Acta Math. 114, 1965, 1-70.

[HKT] Hanson, B., P. Koskela, and M. Troyanov: Boundary behaviour of quasi-regular maps and the isodiametric profile. - Conform. Geom. Dyn. 5, 2001, 81-99.

[Her] Herron, D. A.: Conformal deformations of uniform Loewner spaces. - Math. Proc. Cambridge Philos. Soc. 136, 2004, 325-360.

[KL] Koskela, P., and P. Lammi: Gehring-Hayman theorem for conformal deformations. Preprint 915, CRM Barcelona, 2009, http://www.crm.es/Publications/09/Pr915.pdf.

[Ma] Mattila, P.: Geometry of sets and measures in Euclidean spaces: Fractals and rectifiability. - Cambridge Univ. Press, Cambridge, 1985.

[Mu] Munkres, J. R.: Topology. - Second edition, Prentice-Hall, 2000.

[NT1] Nieminen, T., and T. Tossavainen: Boundary behavior of conformal deformations. Conform. Geom. Dyn. 11, 2007, 56-64.

[NT2] Nieminen, T., and T. Tossavainen: Conformal metrics on the unit ball: The GehringHayman property and the volume growth. - Conform. Geom. Dyn. 13, 2009, 225-231.

[Po] Pommerenke, CH.: Boundary behavior of conformal maps. - Springer-Verlag, Berlin, 1992.

[St] Stein, E. M.: Singular integrals and differentiability properties of functions. - Princeton Math. Ser. 30, Princeton Univ. Press, Princeton, NJ, 1970.

[Va] VÄısÄLÄ, J.: Lectures on $n$-dimensional quasiconformal mappings. - Lecture Notes in Math. 229, Springer-Verlag, Berlin, 1971.

Received 25 January 2010 